\title{
CAMBIOS EN LA POBLACIÓN DE ELCHE ENTRE 1970 Y 1986 UN EJEMPLO DE LA CRISIS DEMOGRÁFICA DEL PAÍS VALENCIANO
}

\author{
Salvador Palazón Ferrando
}

\begin{abstract}
RESUMEN
Elche ha sido durante varias décadas uno de los municipios con demografía más dinámica de la Comunidad Valenciana; sin embargo, en los últimos años no ha podido evitar la crisis de crecimiento que padece actualmente la región. Dos son los factores que han determinado este cambio tan significativo en la demografía ilicitana: la desaparición de la inmigración y el descenso de la tasa de natalidad.

Las causas de ambos fenómenos, los diferentes tipos de crecimiento poblacional que presentan los barrios de la ciudad y las partidas rurales, y sus repercusiones socio-demográficas son algunos de los aspectos tratados en este artículo.
\end{abstract}

\section{RÉSUMÉ}

Elche a été pendant plusieurs décades une des communes avec une démographie des plus dynamiques de la Comunidad Valenciana; mais dans les demières années elle n'a pas pu échapper à la crise de croissance qui subit actuellemente la région. Les causes qui ontdeterminé ce changement si significatif dans la démographie d'Elche sont: la desaparition de l'inmigration et la baisse du taux de natalité.

Les causes de ces phénomènes, les diférentes types de croissance de la population que présentent les quartiers de la ville et les zones rurales, et les rèpercussions socio-démographiques qui s'en derivent sont quelques uns des aspects étudiés dans cet article.

Elche ha sido durante varias décadas uno de los municipios con demografía más dinámica de la Comunidad Valenciana, debido a una masiva inmigración y al crecimiento natural derivado de la misma; sin embargo, en los últimos años no ha podido evitar la crisis de crecimiento que padece actualmente la región ${ }^{1}$, así como buena parte del país.

1 Veáse a este respecto GOZÁLVEZ PÉREZ, V., "La población valenciana en1986: la evidencia de una fuerte y abierta crisis", Cuadernos de Geografia, número 42, pp. 149-158, Universidad de Valencia, 1987. 
Esta crisis, que ya se vislumbra en la segunda mitad de la década de los setenta, se intensifica a partir de 1981; si la población ilicitana entre 1961-1970 y 1971-75 aumentó a un ritmo de crecimiento anual acumulado de 5,3\% y 3,8\%, respectivamente, este ritmo desciende a $2 \%$ en 1976-81, y se reduce a 1,3\% en el primer quinquenio de los ochenta, 1981-86.

Cuadro I

TASAS DE CRECIMIENTO ANUAL ACUMULADO DE ELCHE 1960-1986

\begin{tabular}{ccccc}
\hline AÑo & $\begin{array}{c}\text { POBLACION DE } \\
\text { HECHO }\end{array}$ & $\begin{array}{c}\text { DIFERENCIA CENSAL } \\
\text { ANNO ANTERIOR }\end{array}$ & $\begin{array}{c}\text { AUMENTO } \\
\text { CENSAL } \%\end{array}$ & $\begin{array}{c}\text { T.C.A.A. } \\
\%\end{array}$ \\
\hline 1960 & 73.320 & & & \\
1970 & 122.664 & 49.344 & 67,3 & 5,28 \\
1975 & 147.614 & 24.950 & 20,0 & 3,77 \\
1981 & 162.873 & 15.259 & 10,3 & 1,99 \\
1986 & 173.392 & 10.519 & 6,5 & 1,26 \\
\hline Fuente: I.N.E.: Censo de la Población de España & & \\
AYTO. ELCHE: Padrón Municipal de Habitantes. & & \\
Elaboración propia &
\end{tabular}

Aún así, Elche mantiene durante los años ochenta la tasa de crecimiento más alta entre las principales ciudades de la Comunidad Valenciana, tal como indican las cifras del Cuadro II.

Cuadro II

TASAS DE CRECIMIENTO ANUAL ACUMULADO DE ALGUNAS CIUDADES DE LA COMUNIDAD VALENCIANA EN EL PERIODO 1981-86

\begin{tabular}{|c|c|}
\hline & T.C.A.A. $(\%)$ \\
\hline Alcoy & $-0,14$ \\
\hline 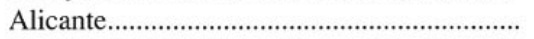 & 1,10 \\
\hline 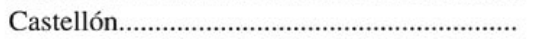 & 0,50 \\
\hline 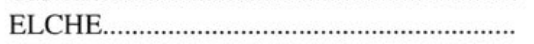 & 1,26 \\
\hline 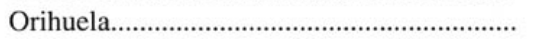 & 1,00 \\
\hline 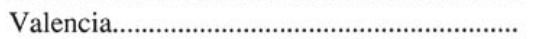 & $-0,30$ \\
\hline
\end{tabular}

Elaboración propia.

Dos son los factores causantes de este cambio tan significativo en la demografía ilicitana: la casi total desaparición de la inmigración y el descenso del crecimiento vegetativo.

\section{La desaparición de la inmigración neta}

La masiva inmigración y sus repercusiones demográficas (elevadas tasas de natalidad y rejuvenecimiento de la población) fueron el motor del elevado crecimiento demográfico ilicitano durante los sesenta y principios de los setenta. Sin embargo, el marcado carácter 


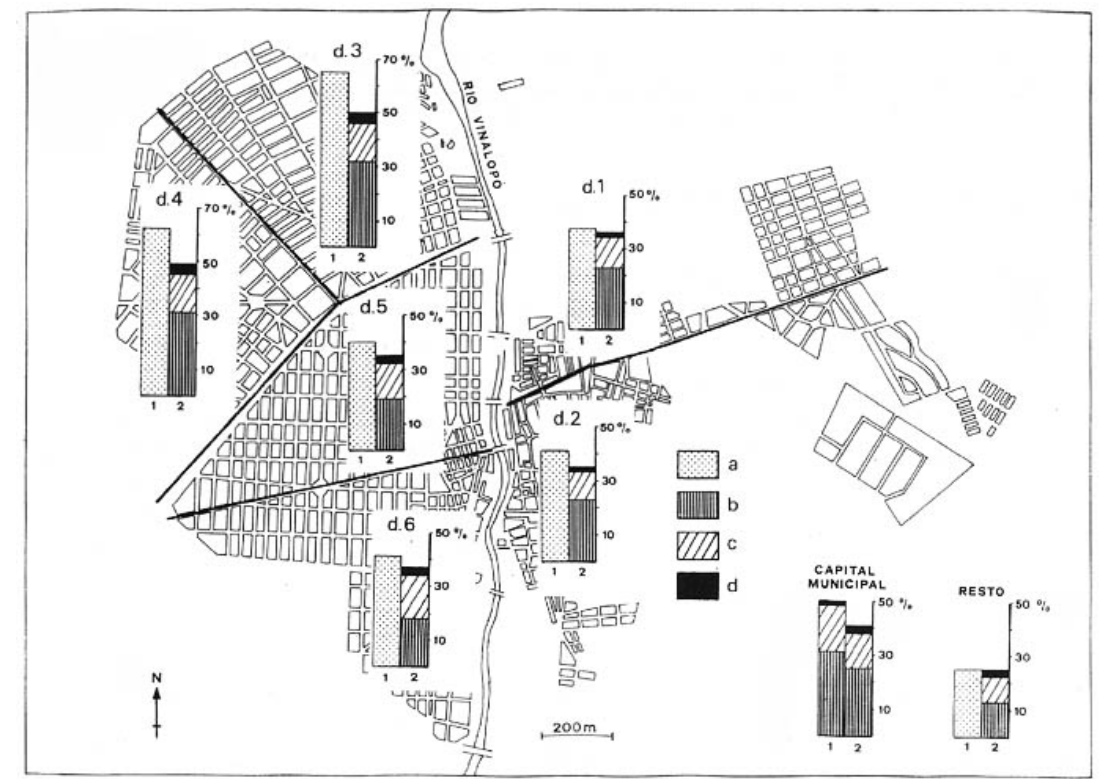

MAPA 1.- Distribución de la inmigración en Elche por distritos urbanos y lugar de procedencia 1, año 1970; 2 , año 1986. Procedencia: a, sin diferenciar; b, extraprovincial; c, provincia de Alicante; d, extranjero. En trazo grueso la división entre distritos.

obrero de esta inmigración, la crisis en la que entra tras 1973 la industria del calzado, principal actividad económica generadora de empleo en el municipio, y la amplia oferta de mano de obra municipal (resultado de la elevada natalidad de los sesenta), han conducido a una extraordinaria reducción del movimiento inmigratorio en los últimos años. De tal forma que si el saldo migratorio positivo representaba el 63\% del crecimiento intercensal durante 1960-70 (31.392 de 49.344 habitantes), para 1976-81 supone sólo el 7,8\% (1.186 de 15.259) y queda convertido en un 4,4\% (435 de 10.519) en 1981-86, es decir, una inmigración casi testimonial (Cuadro III).

Otro hecho que nos indica el bache en el que ha entrado la inmigración, es la pérdida de peso relativo que en 1986 presenta la comunidad inmigrada frente a la autóctona en todos los distritos urbanos con respecto a la situación existente en 1970 (Vid. Mapa 1). La comunidad inmigrada, en 1986, representa un total de 68.645 habitantes (trece mil más que en 1970) y está integrada en un $60 \%$ por gentes procedentes de otras provincias (entre las que destacan Murcia, Albacete, Granada y Jaén), en una tercera parte (32\%) por alicantinos originarios en su mayoría de las comarcas del Bajo Segura, Vinalopó Medio y Campo de Alicante, y el resto (8\%) por personas cuya residencia anterior era el extranjero (en este caso se trata más de emigrantes españoles que retornan a nuestro país que de nacionales de otros países).

Aunque en todos los distritos urbanos hay una presencia importante de inmigrados, 
sobresale Carrús (distritos tercero y cuarto) que absorbe al $47 \%$ del total, debido a que su poblamiento tanto inicial como posterior se hizo básicamente con los llegados de fuera.

\section{El descenso del crecimiento natural}

No solo los bajos saldos migratorios han ocasionado la disminución del aumento demográfico. El crecimiento vegetativo, prácticamente el único responsable del aumento intercensal en los últimos diez años, también ha experimentado una tendencia decreciente, fruto no de un aumento de la mortalidad (prácticamente estancada y con tasas muy bajas dada la juventud de la población ilicitana), sino de la caída de la natalidad, tanto en cifras absolutas (los nacimientos han disminuido en un $20 \%$ entre 1976-81 y 1981-86) como relativas (Vid. Cuadro III)

\begin{tabular}{lcr}
\multicolumn{2}{c}{ Cuadro III } \\
MOVIMIENTO NATURAL $Y$ SALDOS MIGRATORIOS DE ELCHE, & 1976-86 \\
\hline & $\mathbf{1 9 7 6 - 8 1}$ & $\mathbf{1 9 8 1 - 8 6}$ \\
\hline Nacimientos & 18.950 & 15.054 \\
Defunciones & 4.877 & 4.970 \\
Crec. Veget. & 14.073 & 10.084 \\
Saldo Migrat. & +1.186 & +435 \\
Crecim. Real & 15.259 & 10.519 \\
Tasa Nat.* & 24,6 & 18,0 \\
Tasa Mort.* & 6,3 & 5,9 \\
Tasa C.V.* & 18,3 & 12,1 \\
Tasa C.R.* & 19,9 & 12,6 \\
\hline
\end{tabular}

* Tasa media anual. Cifras en tantos por mil.

Fuente: I.N.E.: Movimiento Natural de la Población de España Elaboración Propia.

La causa de la reducción de la natalidad se debe al aumento del control voluntario del embarazo, consecuencia entre otras razones de la inseguridad económica de los jóvenes (que retrasa tanto la edad de matrimoniar como las expectativas de ampliar la familia), el uso creciente y eficaz de los anticonceptivos, el aumento de la actividad profesional de la mujer y su mayor independencia, el consumismo, etc ${ }^{2}$. Sin embargo, Elche continúa teniendo una de las tasas de natalidad más altas del País Valenciano, resultado de la elevada inmigración procedente de la España meridional que llega en la década anterior y cuyos esquemas mentales sobre el tamaño de la familia no han cambiado con la rapidez que lo han hecho los de la población autóctona.

2 GOZÁLVEZ PÉREZ, V., “Dinámica natural de la población española, 1970-1986”, Primeras Jornadas sobre Geografía de la Población Española, 1970-1986, Salamanca, Ed. Síntesis, 1987 (en prensa). 

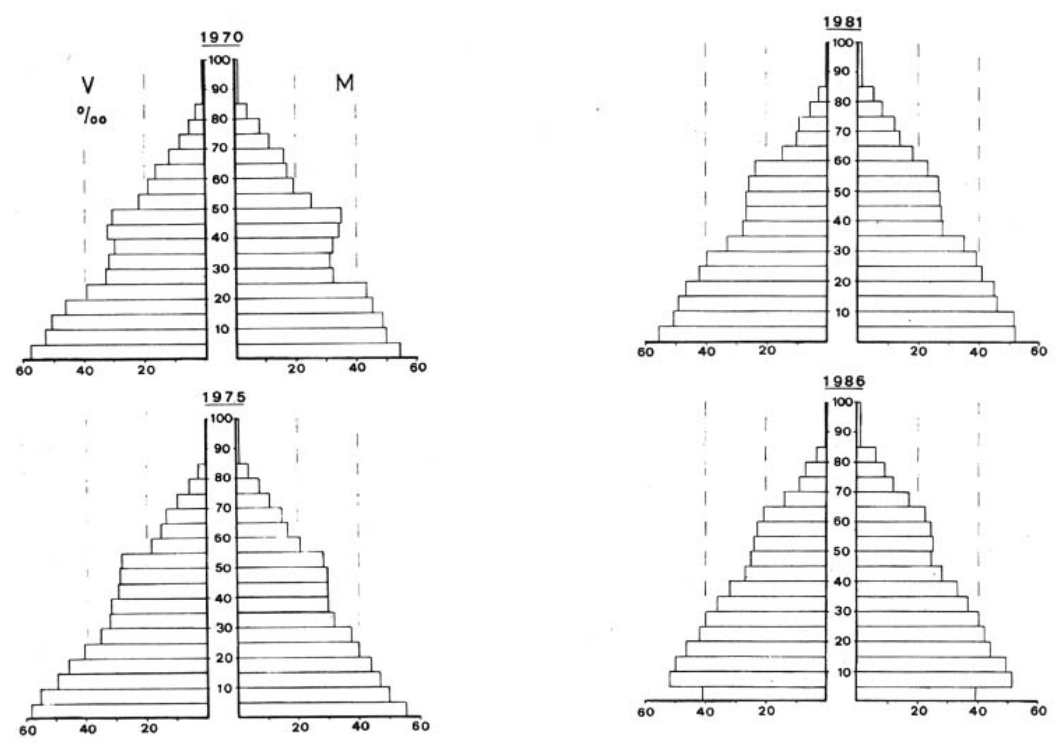

La estructura por edad y sexo de Elche en los últimos quince años (Vid. Figura 1), ofrece claras diferencias entre 1970 y 1986. En 1970 la inmigración y sus consecuencias son claramente visibles en el perfil de la pirámide, tanto por el engrosamiento anormal de los adultos como por el ensanchamiento de la base. Sin embargo, a partir de 1975 las huellas del aporte inmigratorio se perciben con más dificultad en los adultos, mientras que en la base no será hasta 1981 cuando comiencen a apreciarse los resultados del descenso de la natalidad. Este descenso continuado de los nacimientos durante los años ochenta da lugar al brusco estrechamiento de la primera cohorte de la pirámide municipal del año 1986 y al rápido envejecimiento de la población ilicitana; así, si en 1970 y 1975 el índice de vejez es de veintiuna personas mayores de 65 años por cada cien jóvenes menores de 16, y en 1981 este índice es de veintitrés, cinco años después, en 1986, asciende ya a treinta y uno.

\section{EI desigual crecimiento demográfico en el término municipal}

Dentro del municipio se distinguen diferencias de crecimiento no sólo entre el campo y la ciudad sino también dentro de los espacios de cada uno de ellos.

La población rural se halla inmersa desde antes de 1960 en una situación regresiva ( $\mathrm{Vid}$. 
Cuadro IV), consecuencia del escaso aporte inmigratorio recibido (tanto en 1970 como en 1986 los inmigrantes suponen menos del $30 \%$ de la población total), del éxodo rural y del consiguiente envejecimiento demográfico ${ }^{3}$ y descenso de la natalidad, aspectos que quedan plasmados con fuerza en la distribución por sexo y edad, de 1986, tal como se ve en la Figura 2. La pérdida de población es generalizada en todas las partidas rurales ilicitanas, excepto en Torrellano y El Altet que presentan un crecimiento continuado, gracias a la existencia de dos importantes núcleos de población que, por su situación sobre las carreteras nacionales

Cuadro IV

\begin{tabular}{|c|c|c|c|c|}
\hline & 1960 & 1970 & 1981 & 1986 \\
\hline udad & 50.989 & 102.357 & 144.673 & 155.637 \\
\hline impo & 22.331 & 21.392 & 20.106 & 20.012 \\
\hline
\end{tabular}

Fuente: GOZALVEZ PEREZ, V.: La ciudad de Elche y El Bajo Vinalopó

AYTO. ELCHE: Padrón Municipal de Habitantes

340 y 332 , respectivamente, a escasa distancia de la capital municipal y de la provincial, han adquirido las características de núcleos-dormitorio.

Por otra parte, el crecimiento demográfico urbano se ha aminorado en los últimos años ${ }^{4}$, pues mientras durante los sesenta se expansionó en grado extremo debido a las necesidades de mano de obra que exigía la boyante industria del calzado, ésta desde mediados de los setenta pasa a abastecerse casi exclusivamente con la fuerza de trabajo local o de municipios cercanos mediante migraciones pendulares y no definitivas.

La cartografía de las tasas de crecimiento anual acumulado de las secciones padronales urbanas $^{5}$ para 1970-81 y 1981-86 (Vid. Mapas 3 y 4), nos muestra, entre otras, estas características: a) la tendencia general ha sido el descenso de dichas tasas, de tal forma que si en el primer período la mitad de las secciones (de un total de sesenta y una) presentaban incrementos superiores al 3\% y sólo cinco tenían tasas negativas, esta proporción se invierte en el primer quinquenio de los ochenta, pues mientras nueve secciones conservan tasas por encima del 3\%, la mitad de las secciones urbanas presentan incrementos inferiores a $0 \%$; y b) las diferentes secciones pueden agruparse en tres grandes grupos según haya sido su crecimiento anual acumulado: 1) Inferior a $1 \%$ en la década de los setenta y negativo en el

3 El índice de vejez de la población extraurbana es de cuarenta y cuatro personas mayores de 65 años por cada cien jóvenes menores de 16 años, frente a un índice de treinta y uno que presenta la población total ilicitana.

4 La tasa de crecimiento anual acumulado de la población urbana fue de 7,21\% durante el período 1960-70, de 3.52 de entre 1970 y 1981, reduciéndose a 1,47\% para el primer quinquenio de los ochenta.

5 Como en 1970 el casco urbano estaba compuesto por tres distritos y sesenta y una secciones padronales, y en 1986 por seis distritos y 115 secciones, resultado de la división de los primeros, hemos optado, con el fin de poder realizar comparaciones, por agrupar las secciones de 1986 en los perímetros que tenían las de 1970, aunque se mantengan los seis distritos de 1986. 


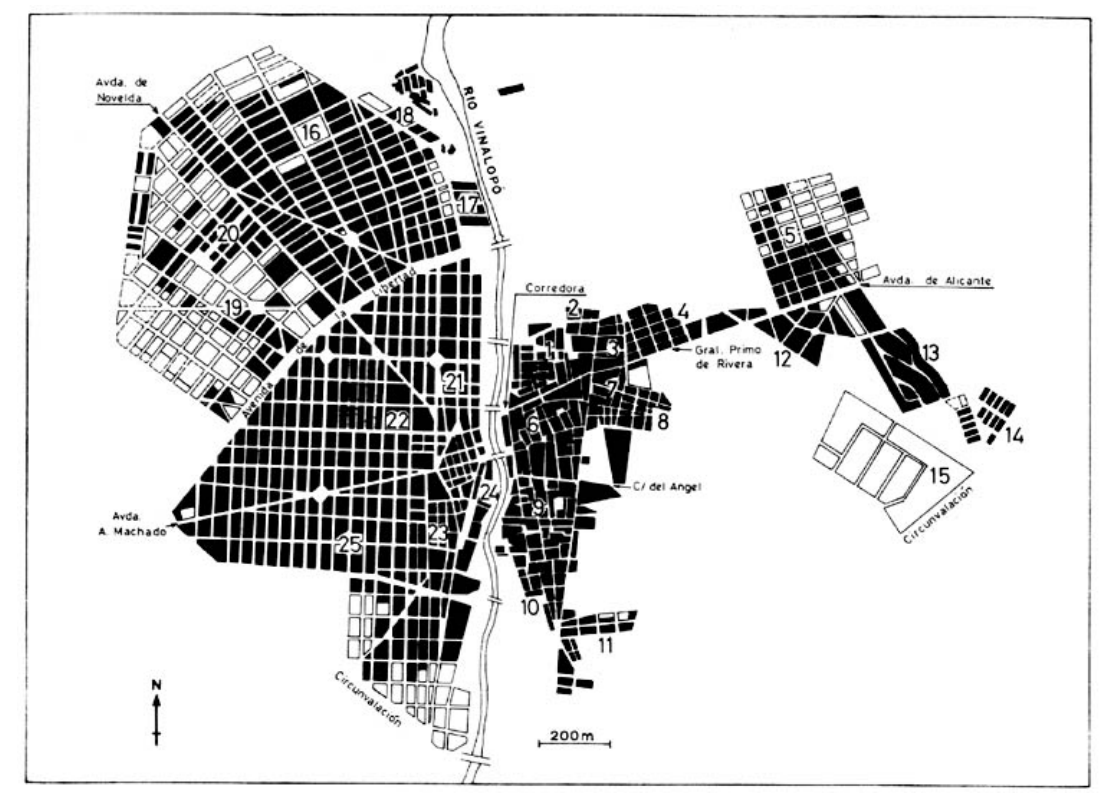

MAPA 2.- Crecimiento urbano de Elche, 1967-1986. En negro la superficie edificada hasta el año 1967; en blanco y trazo continuo la superficie edificada entre 1967 y 1986; en blanco y trazo discontinuo la superficie planificada y no edificada todavía en 1986. Distrito Primero: 1, Vila; 2, Illeta; 3, Barrio Chinchilla; 4, Barrio de la Barrera; 5, Barrio de la Lonja. Distrito Segundo: 6, Barrio del Salvador; 7, Barrios del Carmen y de San Miguel; 8, Barrio les Pataes; 9, Arrabal de San Juan, 10, Barrio de Tripa; 11, Puertas Coloradas. Distrito Tercero: 16, Carrús Este; 17, Barrio La Rata; 18, Barrios de Casablanca, Patilla y Cuevas de Carrús. Distrito Cuarto: 19, Carrús Oeste; 20, Barrio de la Sagrada Familia. Distrito Quinto: 21, Barrio del Pont Nou; 22, Plá de Sant Josep Norte. Distrito Sexto: 23, Barrio del Asilo; 24, Arrabal de Santa Teresa; 25, Plá de Sant Josep Sur.

primer quinquenio de los ochenta; 2) Superior a $1 \%$ en los setenta e inferior a $1 \%$ en los ochenta; y 3 ) Superior a $1 \%$ en los setenta y en los ochenta ${ }^{6}$. Estos tres tipos de crecimiento poblacional están directamente relacionados con el aumento del parque inmobiliario ${ }^{7}$ y de la superficie edificada (Vid. Mapa 2).

Barrios con crecimiento demográfico muy lento o regresivo en los años setenta y regresivo en los ochenta
$\mathrm{L} o$
p r e s e n t a $n$
t a n to
a $1 \mathrm{~g} \mathrm{u} \mathrm{n} \mathrm{o} \mathrm{s}$
b a r r i o s
d e 1

centro

( Vila,

Arrabal

de San

Juan

(al sur

de $1 \mathrm{a}$

c a $11 \mathrm{e}$

6 Hemos optado por usar el 1\% como límite por que todas las secciones con una tasa superior presentan un crecimiento demográfico progresivo, entre $1 \%$ y - 1\% están estancadas, mientras las inferiores a $-1 \%$ muestran un decremento poblacional.

7 Para estudiar el crecimiento del parque inmobiliario se ha utilizado el Censo de Viviendas de los años 1970 y 1981 y se ha realizado, ante la falta de un recuento oficial en 1986, una estimación usando la Relación de Licencias de Obras concedidas entre 1980 y 1984, ambos inclusive, existente en el Negociado de Obras y Urbanismo del Ayuntamiento de Elche y donde se especifican las construcciones de nuevos edificios; estas obras han sido contabilizadas por secciones según la calle en que han de realizarse, estableciendo un coeficiente de dos viviendas por planta. 


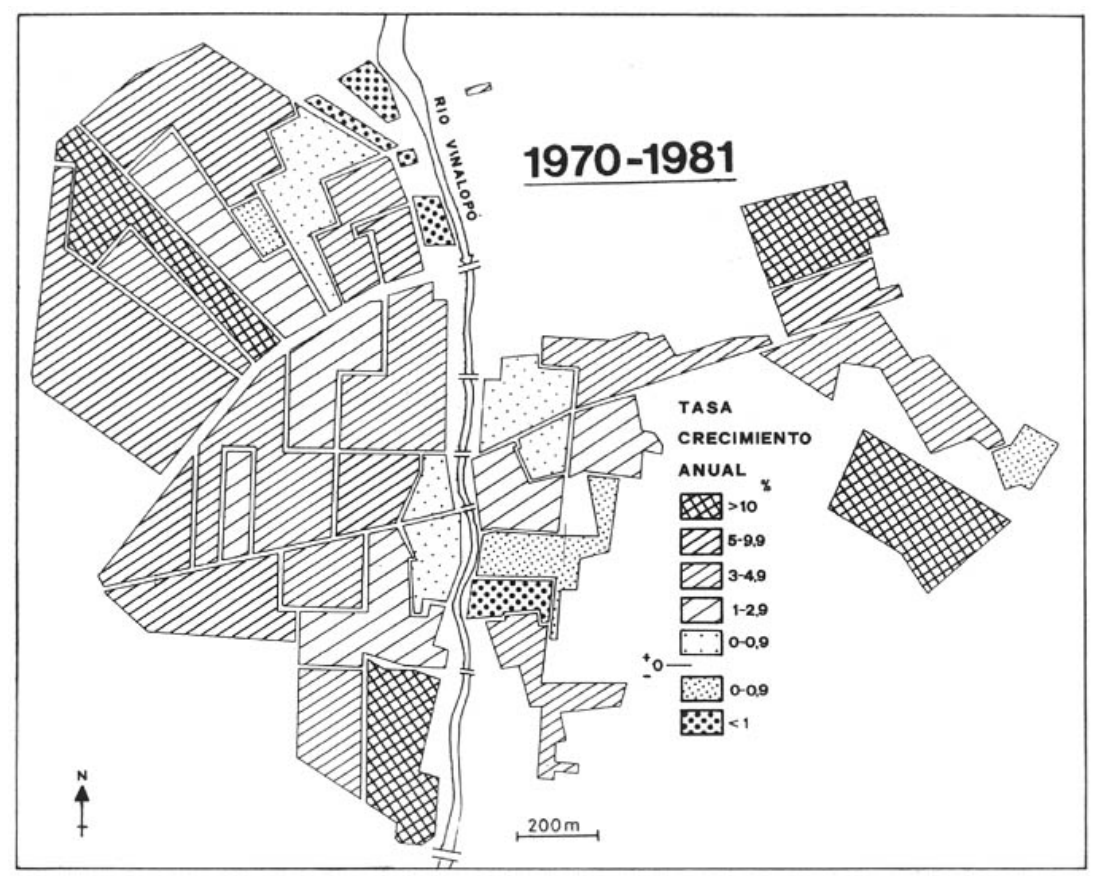

MAPA 3.- Distribución de las tasas de crecimiento anual acumulado por secciones en la ciudad de Elche, 1970-1981.

del Ángel), Salvador y Arrabal de Santa Teresa), como aquellos barrios o zonas que absorbieron la inmigración masiva de principios de los años sesenta (Casablanca, La Rata, San Antón y buena parte de Carrús Este ${ }^{8}$ ).

En todos estos barrios el principal problema ha radicado en la dificultad para conseguir en ellos nuevas viviendas desde finales de los sesenta; el resultado ha sido: la "expulsión" de una parte de la población joven (sobre todo en los barrios de inmigrantes donde abundan las familias numerosas con hijos mayores de edad) e imposibilitar, prácticamente, el asentamiento de nuevos vecinos, obligándolos a fijar su residencia en otras zonas de la ciudad, con la consiguiente pérdida efectiva de población, un envejecimiento de la que se queda y un descenso de la natalidad por disminución de la población en edad de procrear (descenso que no hay que olvidar también se debe a un mayor control del embarazo como ya se ha dicho).

8 Carrús es la zona que se halla en la margen derecha del Vinalopó al norte de la Avenida de la Libertad (antiguo trazado del ferrocarril) y está dividida en dos sectores, este y oeste, por la Avenida de Novelda que lo cruza de norte a sur. 


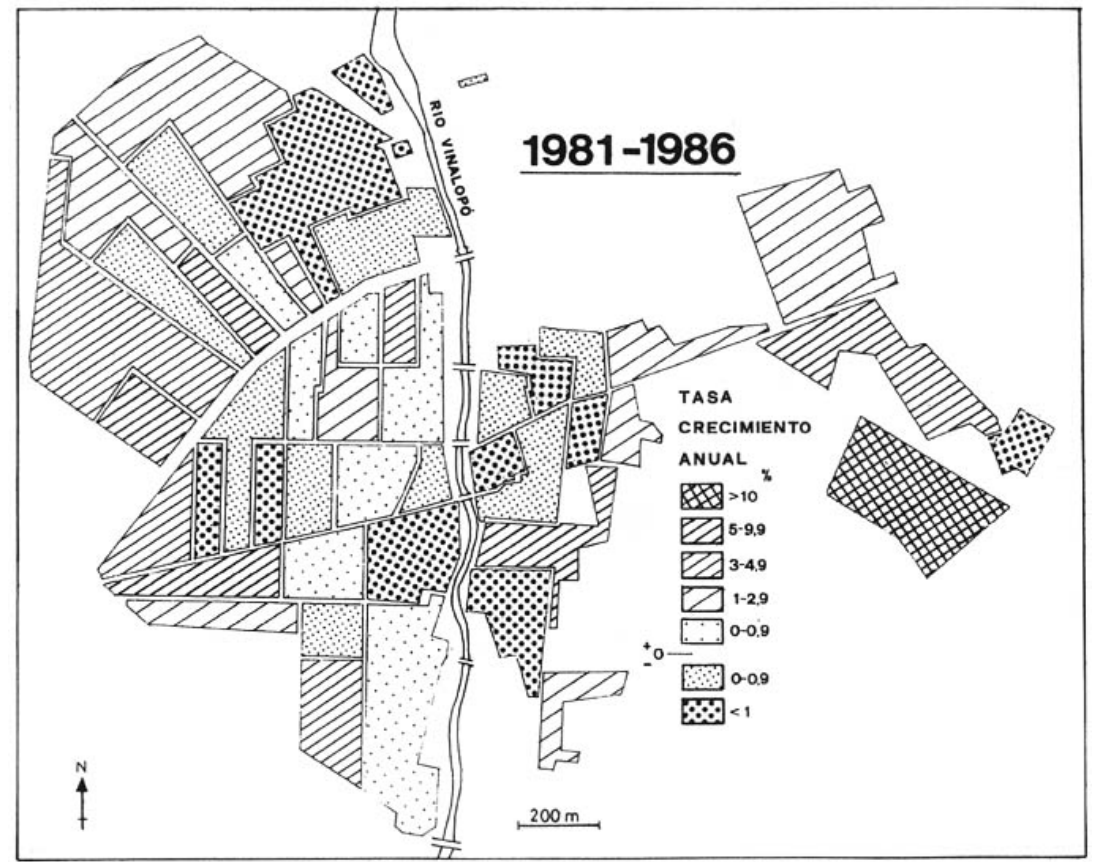

MAPA 4.- Distribución de las tasas de crecimiento anual acumulado por secciones en la ciudad de Elche, 1981-1986.

Las causas que dificultan el acceso a nuevas viviendas varía de unos barrios a otros:

-En la Vila y el Salvador radica en el elevado precio que adquieren las viviendas en esta zona por sus características céntricas.

-En el Arrabal de San Juan es debido a los Planes Especiales a los que se le ha sometido durante años y que limita tanto el número de construcciones como la alturas de las misma ${ }^{9}$.

-En el Arrabal de Santa Teresa por la proliferación de viviendas tradicionales (casas de planta baja y uno o dos pisos) que no han sido sustituidas por bloques de viviendas.

- En San Antón, Casablanca y La Rata por ser barrios de viviendas sociales en bloque abierto y de número limitado.

9 Como resultado del Plan Especial incluido en el P.G.O.U. de 1973, las Normas Urbanísticas limitaron la altura de los nuevos edificios a tres plantas en los solares sin edificar o a la altura del edificio que fuera a sustituirse. De ahí que mientras la Vila ha seguido una práctica de adaptación y revalorización a las nuevas condiciones y exigencias del Centro y de sus propietarios, en el Arrabal las políticas emprendidas han generado aún mayores niveles de expulsión, como señala SEVILLA, M., Crecimiento y Urbanización. Elche 1960-1980, Valencia, Ayto. de Elche, 1985. Si en el mapa 3, una parte del Arrabal parece ganar población entre 1981 y 1986 , se debe a que está incluida en la misma sección padronal que un huerto de la Puerta Morera, ocupado recientemente por un conjunto residencial que no participa del proceso de degradación de aquél por quedar fuera de sus límites. 


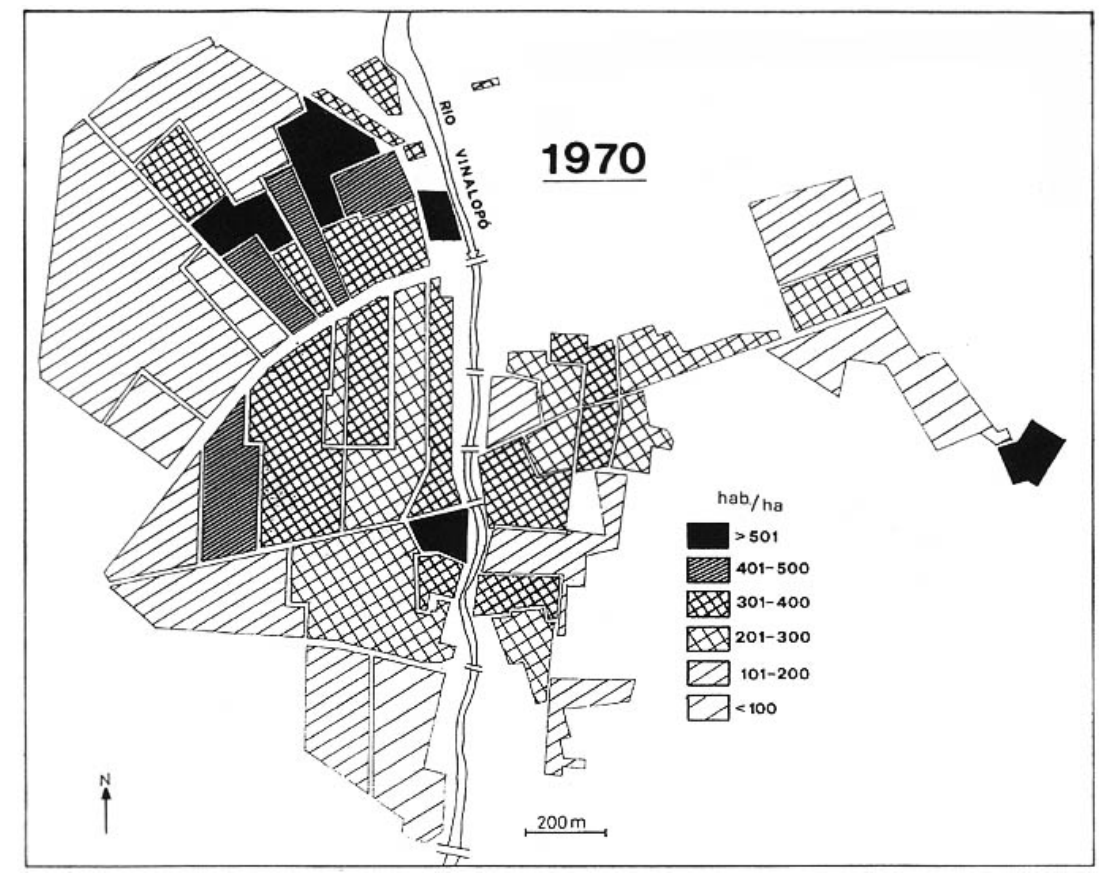

MAPA 5.- Densidades demográficas por secciones en la ciudad de Elche, año 1970.

-En Carrús Este por la rápida y casi total ocupación del espacio en los años sesenta por edificios de elevada altura que reducen las posibilidades de realizar nuevas construcciones posteriormente. ${ }^{10}$

\section{Crecimiento demográfico durante los setenta y total paralización en los ochenta}

Se da en los barrios de Chinchilla, Illeta, Tripa, San Miguel, Carmen, Pont Nou, Asilo y Arrabal de San Juan (Norte de la Calle del Ángel), y también afecta a gran parte del Plá de Sant Josep, al sector occidental de Carrús Este y a la zona central de Carrús Oeste, como resultado de heredar con unos años de diferencia la inadecuación entre población y oferta de viviendas libres que se daba anteriormente en los barrios ya citados (Vila, Salvador, Casablanca, etc). El retraso estriba en que el aumento más tardío del parque inmobiliario en estas zonas, basado en una sustitución de casas de planta baja y/o uno o dos pisos por edificios de varias plantas, les permitió absorber en un primer momento (década de los setenta) sus propios excedentes, así como los procedentes de otros barrios y parte de la inmigración

10 En Casablanca, La Rata, San Antón y Carrús Este la ocupación de viviendas fue muy rápida porque absorbió gran parte de la masiva inmigración de los primeros años sesenta. 


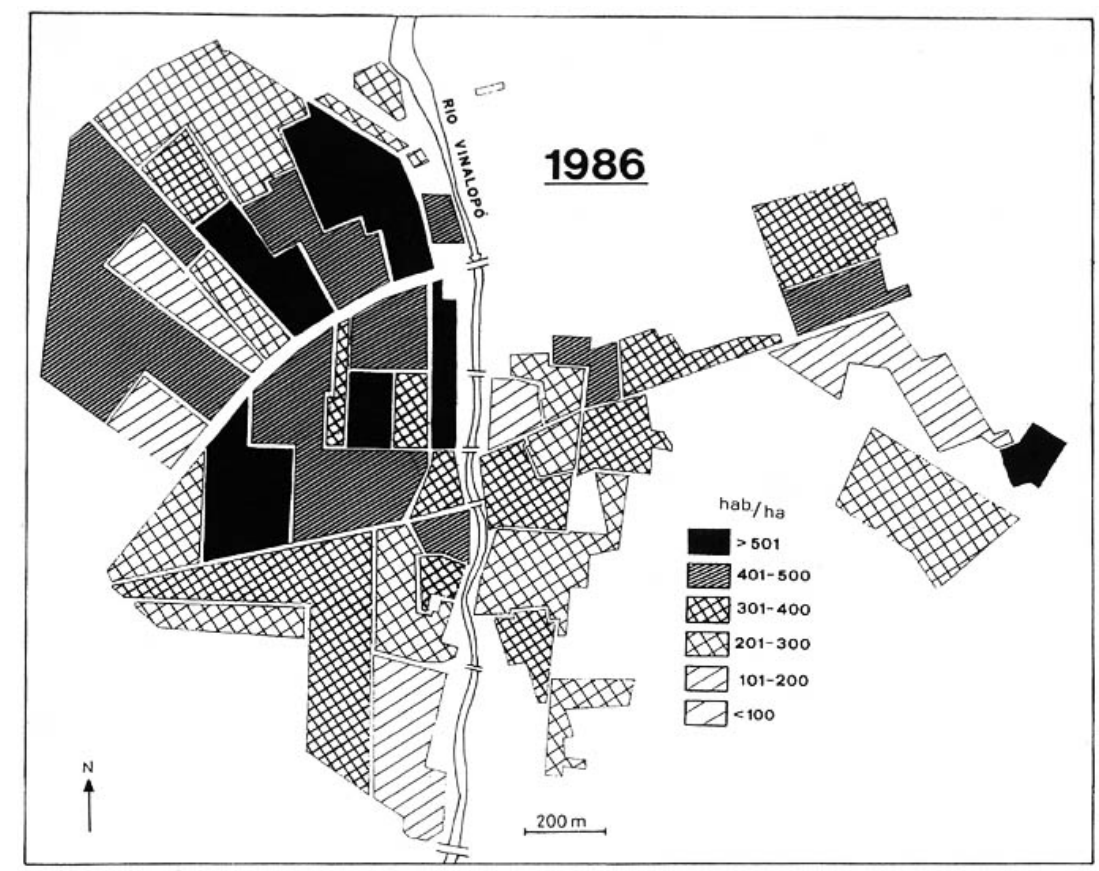

MAPA 6.- Densidades demográficas por secciones en la ciudad de Elche, año, 1986.

llegada en la segunda mitad de la década de los sesenta y principios de los setenta; sin embargo, posteriormente, dado sus elevados crecimientos demográficos, se producirá el desajuste población-viviendas libres y dará comienzo el "éxodo" hacia otros barrios o zonas con capacidad para aceptar más población.

Ahora bien, ese desajuste no está provocado por el agotamiento de los factores que permitieron su crecimiento inmobiliario, pues en todos los barrios o sectores queda un buen número de casas de planta baja y/o uno o dos pisos susceptibles de ser sustituidas por edificios más altos; el citado desajuste debe atribuirse, por tanto, a un interés de los constructores por edificar en los barrios periféricos donde el precio de los solares es más bajo y las posibilidades especulativas mayores. Una excepción a este esquema lo representa la zona central de Carrús Oeste, donde las nuevas construcciones se han visto limitadas por la importante extensión superficial dedicada a usos comerciales y escolares y la existencia de un barrio social (Sagrada Familia).

Crecimiento demográfico continuado desde 1970

Se produce en aquellos barrios o zonas de la periferia (Torreta Verdi, Lonja, Pataes, Puertas Coloradas, Barrera, Carrús Oeste, norte de Carrús Este, y oeste-suroeste del Plá de 

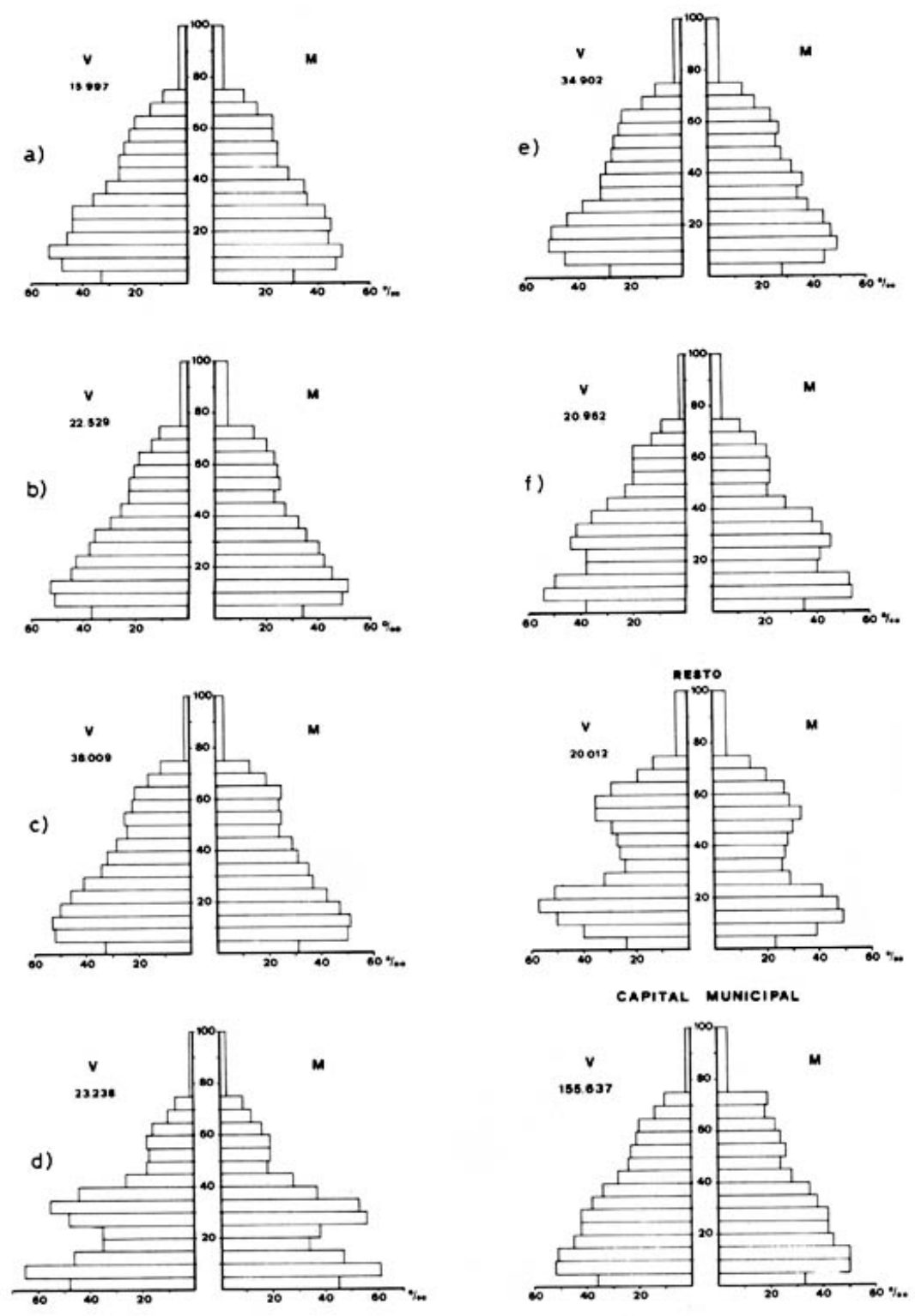

FIGURA 2.- Distribución por sexo y edad de la población de Elche en 1986 según el distrito de residencia. a, distrito primero; $b$, distrito segundo; c, distrito tercero; d, distrito cuarto; e, distrito quinto; f, distrito sexto. 
Sant Josep) donde, por las razones ya aducidas, no solo crecen las posibilidades edificatorias en altura sino también en superficie, ya sea por la ocupación de solares hasta entonces no edificados o bien por la sustitución de la casa tradicional y/o de edificios destinados a actividades industriales por otros dedicados a viviendas, lo que conlleva que estos barrios o sectores urbanos dupliquen, tripliquen e incluso, en algunos casos, quintupliquen en 1986 su parque inmobiliario con respecto a 1970, provocando un fuerte cambio del paisaje urbano entre un año y otro. Este gran incremento de la oferta inmobiliaria les permite absorber los excedentes demográficos que los otros barrios de la ciudad se ven obligados a "expulsar" y será lo que determine su crecimiento continuado en los últimos quince años.

Por otra parte, la elevada tasa de crecimiento anual acumulado (superior al 50\%) que presenta la población de Los Palmerales se debe a ser un barrio de viviendas sociales de bloque abierto construido en una zona de vacío urbano a finales de los setenta y ocupado en muy pocos años por la gran demanda existente de esta clase de viviendas.

El resultado de estos tres tipos de crecimiento demográfico en el plano urbano de Elche repercuten:

a) En un cambio de las densidades demográficas de las secciones padronales entre 1970 y $1986^{11}$ ( Vid. Mapas 5 y 6); todos los barrios o zonas con tasas de crecimiento anual acumulado superior a $1 \%$ durante los años setenta y/o los ochenta han aumentado sus índices en 1986 con respecto a 1970; en cambio, los barrios con incrementos anuales inferiores a 1\%, como resultado de una pérdida efectiva de población, han experimentado un descenso de sus densidades demográficas.

b) En la distribución de la población dentro del casco urbano, al consolidarse en la actualidad la situación existente en 1970, es decir, en la margen derecha del Vinalopó continúa residiendo un $25 \%$ de los ilicitanos y en la margen izquierda el restante $75 \%$.

c) En la estructura por sexo y edad de los diferentes distritos urbanos (Vid. Figura 2). En todos ellos se patentiza la caída de la natalidad en los últimos años, ahora bien, mientras en la mayoría este descenso se produce desde 1975 (ya que los grupos de menos de diez años son inferiores a los de 10 a 14), en los distritos cuarto y sexto tal descenso no es visible hasta 1981, como consecuencia del rejuvenecimiento de su población por inmigración de adultos jóvenes (que aumentan en un principio la natalidad y retrasan su caída ${ }^{12}$ ). Otro resultado del rejuvenecimiento demográfico de los distritos cuarto (Carrús Oeste) y sexto (Sur del Plá de Sant Josep, Arrabal de Santa Teresa y Asilo) son los índices de vejez que presentan, dieciocho y veintisiete $^{13}$, respectivamente, los más bajos de la ciudad (el índice medio urbano es de veintinueve). Por otra parte, los demás distritos urbanos presentan un envejecimiento más acusado, con índices superiores a la media urbana e incluso a la media municipal; únicamente

11 Aunque V. Gozálvez en La ciudad de Elche ya realizó un estudio de las densidades demográficas de la ciudad en 1970, la necesidad de adecuar en lo posible la comparación entre las densidades de 1970 y de 1986 , nos ha llevado a utilizar como área planimetrada para ambas fechas la superficie urbanizada en 1986, y no la usada por Gozálvez.

12 Este hecho corrobora nuestra hipótesis de que se trata de barrios que absorben gente joven de otras zonas de Elche con problemas para conseguir vivienda en las cercanías de su antigua residencia familiar (por la carestía de la vivienda o por carencia de oferta).

13 El mayor índice de vejez del distrito sexto con respecto al cuarto se debe a que el primero de ellos aglutina a barrios que se han estancado demográficamente (Arrabal de Santa Teresa, Asilo, etc.), mientras que el segundo presenta un crecimiento poblacional continuado. 
el distrito tercero (Carpís este), dónde los efectos de la elevada natalidad derivada de la inmigración que absorbe a finales de los años sesenta y principios de los setenta aún se dejan sentir, presenta un índice de vejez similar a la media urbana. 